04.2

\title{
Одномерное квазилинейное уравнение для описания генерации токов увлечения в плазме токамака геликонами
}

\author{
(ㄱ) А.Ю. Попов, Е.З. Гусаков
}

Физико-технический институт им. А.Ф. Иофффе РАН, Санкт-Петербург, Россия

E-mail: a.popov@mail.ioffe.ru

Поступило в Редакцию 17 сентября 2021 г.

В окончательной редакции 1 октября 2021 г.

Принято к публикации 1 октября 2021г.

\begin{abstract}
Получено квазилинейное уравнение, которое позволяет описать эволюцию функции распределения электронов и генерацию токов увлечения под действием быстрой моды промежуточного частотного диапазона (геликона). Показано, что в анализируемом случае уравнение Фоккера-Планка может быть приближенно сведено к одномерному в пространстве продольных скоростей электронов, в котором коэффициент диффузии пропорционален мощности, поглощаемой при взаимодействии частиц с волнами, фазовая скорость которых равна проекции скорости частиц на направление магнитного поля.
\end{abstract}

Ключевые слова: генерация тока, токамак, электромагнитные волны, геликон, квазилинейная диффузия.

DOI: 10.21883/PJTF.2022.02.51916.19028

В настоящее время актуальной задачей является разработка эффективных методов стационарной безындукционной генерации токов увлечения в плазме токамака термоядерных параметров. Наиболее перспективной считается возможность поддержания тока с помощью быстрой моды с частотой, много меньшей частоты нижнего гибридного резонанса, но существенно большей частоты ионного циклотронного резонанса [1]. Использование этой волны - геликона - позволит уменьшить влияние линейного взаимодействия с ионами и нелинейных (параметрических) эффектов. Для описания генерации тока геликонами требуется анализ квазилинейной эволюции функции распределения электронов в результате резонансного взаимодействия с этими волнами. Последняя задача (задача взаимодействия волн и частиц) очень обширная и часто встречается в физике плазмы и астрофизике [2]. В частных случаях генерации тока и взаимодействия волны накачки с альфа-частицами при нижнегибридном нагреве [3] и генерации тока электронными циклотронными волнами [4] общее уравнение в частных производных по проекциям скорости, описывающее квазилинейную диффузию, редуцируется до одномерного уравнения. В случае распространения медленной продольной волны коэффициент диффузии этого уравнения пропорционален мощности, теряемой на магнитной поверхности в результате резонансного взаимодействия волнами, продольная фазовая скорость которых совпадает с продольной скоростью частиц [5]. Это обстоятельство значительно упрощает расчет профиля плотности генерируемого тока, поскольку не требует знания структуры высокочастотных полей в плазме токамака и позволяет ограничиться при исследовании волновой части задачи применением метода лучевых траекторий [6]. К сожалению, до сих пор отсутствует обоснование такого подхода для случая быстрой моды промежуточного частотного диапазона (геликона), которое необходимо для применения эффективных кодов [6], разработанных для описания генерации токов в плазме токамака медленной модой промежуточного частотного диапазона, при планировании экспериментов с геликонами. В настоящей работе мы восполним этот пробел и получим соответствующее одномерное квазилинейное кинетическое уравнение.

Рассмотрим пакет электромагнитных волн промежуточного диапазона частот $\omega_{c i} \ll \omega<\omega_{L H} \ll\left|\omega_{c e}\right|$ $\left(\omega_{L H}=\omega_{p i} / \sqrt{1+\omega_{p e}^{2} / \omega_{c e}^{2}}-\right.$ нижнегибридная частота, $\omega_{p i, p e}, \omega_{c i, c e}$ - ионная и электронная плазменные и циклотронные частоты), который распространяется под углом к внешнему магнитному полю $\mathbf{B}=B e_{z}$ в однородной плазме:

$$
\mathbf{E}_{0}(\mathbf{r})=\int_{-\infty}^{\infty} \frac{d k_{z}}{4 \pi} \mathbf{A}\left(k_{z}\right) \exp \left(i k_{x}\left(k_{z}\right) x+i k_{z} z-i \omega t\right)+c . c .
$$

где $\mathbf{A}=\mathbf{e}_{G} A_{0}, A_{0}$ - амплитуда,

$$
\mathbf{e}_{G}=\left(1,-i g /\left(n_{z}^{2}+n_{x}^{2}-\varepsilon\right), n_{x} n_{z} /\left(n_{x}^{2}-\eta\right)\right)
$$

- компоненты вектора поляризации,

$$
\varepsilon=1+\frac{\omega_{p e}^{2}}{\omega_{c e}^{2}}-\frac{\omega_{p i}^{2}}{\omega^{2}}<0, \eta=-\frac{\omega_{p e}^{2}}{\omega^{2}}<0
$$

- диагональные, а $g=\frac{\omega_{p e}^{2}}{\omega_{c e} \omega} \gg 1-$ недиагональный компонент тензора диэлектрической проницаемости „холодной“ плазмы,

$$
\begin{gathered}
n_{x}=c k_{x} / \omega=\sqrt{\left(g^{2} /\left(n_{z}^{2}-\varepsilon\right)\right)-\left(n_{z}^{2}-\varepsilon\right)} \approx g / n_{z}, \\
n_{z}=k_{z} c / \omega
\end{gathered}
$$

- компоненты коэффициента преломления. Рассмотрим далее кинетическое уравнение для однородной замагни- 
ченной плазмы, которое описывает функцию распределения электронов [2]:

$$
\begin{aligned}
& \left(\frac{\partial}{\partial t}+v_{i} \frac{\partial}{\partial x_{i}}-\frac{|e|}{m_{e}}\left(E_{i}+e_{i j k} v_{j} B_{k}\right) \frac{\partial}{\partial v_{i}}\right. \\
& \left.-\omega_{c} e_{i j z} v_{j} \frac{\partial}{\partial v_{i}}\right) f_{e}=\operatorname{St}\left(f_{e}\right),
\end{aligned}
$$

где $e_{i j z}-$ полностью антисимметричный единичный тензор, $\operatorname{St}\left(f_{e}\right)$ - столкновительный интеграл в форме Ландау. Будем искать решение уравнения (2) в виде $f_{e}=n f_{0}+f^{(1)}$, где $n-$ плотность плазмы, $f_{0}-$ квазиравновесная, не зависящая от гироугла вращения частиц функция распределения, $f^{(1)}=(2 \pi)^{-1} \int_{-\infty}^{\infty} d k_{z} f^{(1)}\left(k_{z}\right)-$ ее линейная поправка. Подставим это разложение в (2) и получим уравнения для парциальной линейной поправки к функции распределения, частота и волновое число которой навязаны полем волны:

$$
\begin{aligned}
& \left(-i \alpha+i \lambda \cos \theta+\frac{\partial}{\partial \theta}\right) f^{(1)}\left(k_{z}\right)-\frac{n|e|}{2 m_{e} \omega_{c}} \\
& \times\left(\mathbf{A}\left(k_{z}\right)+\frac{\mathbf{v} \times\left(\mathbf{k} \times \mathbf{A}\left(k_{z}\right)\right)}{\omega}\right) \frac{\partial f_{0}}{\partial \mathbf{v}}=0,
\end{aligned}
$$

где $\alpha=\left(\omega-k_{z} v_{z}\right) / \omega_{c}, \lambda=k_{x} v_{\perp} / \omega_{c}, \omega_{c}=\left|\omega_{c e}\right|, \theta-$ азимутальный угол цилиндрической системы координат $\left(v_{\perp}, \theta, v_{z}\right)$ в пространстве скоростей. Проинтегрировав уравнение (3) и воспользовавшись представлением $\exp (i \lambda \sin \theta)=\Sigma_{p} J_{p}(\lambda) \exp (i p \theta)$, найдем линейную поправку к функции распределения электронов

$$
\begin{aligned}
& f^{(1)}=\int_{-\infty}^{\infty} \frac{d k_{z}}{2 \pi} f^{(1)}\left(k_{z}\right)=i \frac{n|e|}{2 m_{e} \omega_{c}} \\
& \times \int_{-\infty}^{\infty} \frac{d k_{z}}{2 \pi} \sum_{p} \frac{\exp (i p \theta-i \lambda \sin \theta)}{\alpha-p} \mathbf{a}_{p}\left(k_{z}\right) \cdot \mathbf{A}\left(k_{z}\right),
\end{aligned}
$$

где компоненты $\mathbf{a}_{p}$ равны

$$
\begin{gathered}
\left(a_{x p}, a_{y p}\right)=\left(J_{p}^{+}(\lambda),-i J_{p}^{-}(\lambda)\right) \\
\times\left(\left(1-\frac{k_{z} v_{z}}{\omega}\right) \frac{\partial}{\partial v_{\perp}}+\frac{k_{z} v_{\perp}}{\omega} \frac{\partial}{\partial v_{z}}\right) f_{0} \\
a_{z p}=\left(J_{p}(\lambda) \frac{\partial}{\partial v_{z}}+\frac{p J_{p}(\lambda)}{\lambda}\left(\frac{k_{x} v_{z}}{\omega} \frac{\partial}{\partial v_{\perp}}-\frac{k_{x} v_{\perp}}{\omega} \frac{\partial}{\partial v_{z}}\right)\right) f_{0}, \\
J_{n}^{+}(\lambda)=n J_{n}(\lambda) / \lambda, \quad J_{n}^{-}(\lambda)=J_{n}^{\prime}(\lambda) .
\end{gathered}
$$

Для учета резонансного взаимодействия Ландау с электронами в бесконечном ряду по номерам электронных циклотронных гармоник в (4) достаточно удержать нулевой член $p=0$ :

$f^{(1)}=i \frac{n|e|}{2 m_{e} \omega_{c}} \int_{-\infty}^{\infty} \frac{d k_{z}}{2 \pi} \frac{\exp (-i \lambda \sin \theta)}{\alpha} \mathbf{b}\left(k_{z}\right) \cdot \mathbf{A}\left(k_{z}\right) \frac{\partial}{\partial v_{z}} f_{0}$, где вектор $\mathbf{b}$ имеет компоненты $\mathbf{b}=\left[0, i J_{1}(\lambda) k_{z} v_{\perp} / \omega, J_{0}(\lambda)\right]_{\omega=k_{z} v_{z}}, \quad$ в которых учтены только резонансные члены при $\omega=k_{z} v_{z}$. Подставим (5) в (2)

$$
\begin{aligned}
& \left(\frac{\partial}{\partial t}+v_{i} \frac{\partial}{\partial x_{i}}-\omega_{c} e_{i j z} v_{j} \frac{\partial}{\partial v_{i}}\right) n f_{0}-\frac{|e|}{2 m_{e}} \\
& \times \int_{-\infty}^{\infty} \frac{d k_{z}}{2 \pi}\left(\mathbf{A}\left(k_{z}\right)+\frac{\mathbf{v} \times\left(\mathbf{k} \times \mathbf{A}\left(k_{z}\right)\right)}{\omega}\right) \frac{\partial f^{(1)}\left(k_{z}\right)^{*}}{\partial \mathbf{v}} \\
& -\frac{|e|}{2 m_{e}} \int_{-\infty}^{\infty} \frac{d k_{z}}{2 \pi}\left(\mathbf{A}\left(k_{z}\right)^{*}+\frac{\mathbf{v} \times\left(\mathbf{k} \times \mathbf{A}\left(k_{z}\right)^{*}\right)}{\omega}\right) \frac{\partial f^{(1)}\left(k_{z}\right)}{\partial \mathbf{v}} \\
& =\operatorname{St}\left(n f_{0}\right) .
\end{aligned}
$$

Усредним левую и правую части (6) по азимутальному углу, используем формулу Сохоцкого $\left(\omega-k_{z} v_{z}\right)^{-1}=P\left(\omega-k_{z} v_{z}\right)^{-1}-i \pi \delta\left(\omega-k_{z} v_{z}\right)$, где $\delta(\ldots)$ - дельта-функция, усредним по случайной фазе $\left\langle\left(\mathbf{b} \cdot \mathbf{A}\left(k_{z}\right)\right)^{*}\left(\mathbf{b} \cdot \mathbf{A}\left(k_{z}^{\prime}\right)\right)\right\rangle=2 \pi\left|\mathbf{b} \cdot \mathbf{A}\left(k_{z}\right)\right|^{2}$ и получим уравнение квазилинейной диффузии функции распределения электронов из-за их взаимодействия с электромагнитной волной (геликонами) в присутствии столкновений

$$
\begin{aligned}
& \frac{\partial}{\partial t} f_{0}-\frac{\omega_{p e}^{2}}{16 \pi n m_{e}} \frac{\partial}{\partial v_{z}}\left(\int_{-\infty}^{\infty} \frac{d k_{z}}{2 \pi}\left|\mathbf{b} \cdot \mathbf{A}\left(k_{z}\right)\right|^{2}\right. \\
& \left.\times \delta\left(\omega-k_{z} v_{z}\right)\right) \frac{\partial}{\partial v_{z}} f_{0}=\operatorname{St}\left(f_{0}\right) .
\end{aligned}
$$

Видно, что в случае взаимодействия электронов с волной промежуточного диапазона частот это одномерное уравнение по продольным скоростям частиц, в котором коэффициент диффузии зависит от поперечной скорости. Тем не менее предположим, что функция распределения факторизуется, оставаясь максвелловской по поперечным скоростям, т. е. $f_{0}=f_{M}\left(v_{\perp}\right) f_{0 z}\left(v_{z}\right)$, что соответствует случаю сильной диффузии, приводящей к образованию „плато“. По отношению к (7) выполним операцию $\int_{0}^{\infty} \ldots v_{\perp} d v_{\perp}$

$$
\begin{gathered}
\frac{\partial}{\partial t} f_{0 z}-\frac{\partial}{\partial v_{z}} \int_{-\infty}^{\infty} \frac{d k_{z}}{2 \pi} D_{z z}\left(k_{z}\right) \delta\left(v_{z}-v_{f}\right) v_{f} \frac{\partial}{\partial v_{z}} f_{0 z}=\operatorname{St}\left(f_{0 z}\right), \\
D_{z z}\left(k_{z}\right)=\frac{\omega_{p e}^{2}}{4 n m_{e} v_{t e}^{2} \omega} \int_{0}^{\infty}\left|\mathbf{b} \cdot \mathbf{A}\left(k_{z}\right)\right|^{2} \exp \left(-\frac{v_{\perp}^{2}}{v_{t e}^{2}}\right) v_{\perp} d v_{\perp} .
\end{gathered}
$$

Для того чтобы выяснить физический смысл полученного коэффициента диффузии, рассмотрим величину удельных потерь пучка волн (1) в плазме в результате резонанса Ландау, которая равна $Q=(2 \pi)^{-1} \int_{-\infty}^{\infty} d k_{z} Q\left(k_{z}\right)$, 
где $Q\left(k_{z}\right)=\left\langle A_{m}^{*}\left(k_{z}\right) j_{m}\right\rangle /(8 \pi)-$ парциальный вклад компоненты поля $k_{z}, \mathbf{j}=-|e| \int \mathbf{v} f^{(1)} d \mathbf{v}-$ плотность тока электронов в поле волны. В результате получим

$$
\begin{aligned}
Q\left(k_{z}\right) & =\left.\frac{\omega_{p e}^{2} \omega}{4 v_{t e}^{2} k_{z}^{2}} \frac{\partial f_{0 z}}{\partial v_{z}}\right|_{\omega / k_{z} 0} \int_{0}^{\infty}\left|\mathbf{b} \cdot \mathbf{A}\left(k_{z}\right)\right|^{2} \exp \left(-\frac{v_{\perp}^{2}}{v_{t e}^{2}}\right) v_{\perp} d v_{\perp} \\
& =\frac{\varepsilon_{y y}^{\prime \prime}\left|A_{y}\right|^{2}+\varepsilon_{y z}^{\prime \prime} \operatorname{Im}\left(A_{y}^{*} A_{z}\right)+\varepsilon_{z z}^{\prime \prime}\left|A_{z}\right|^{2}}{8 \pi}
\end{aligned}
$$

Последний член в правой части (10) описывает затухание Ландау, первый - магнитную накачку, второй представляет собой интерференционный член [7]. Сравним (10) с (9) и отметим, что парциальный коэффициент диффузии (9) в уравнении (8) пропорционален парциальному вкладу $\mathbf{A}\left(k_{z}\right)$ в величину удельных потерь:

$$
D_{z z}\left(k_{z}\right)=Q\left(k_{z}\right) /\left|n m_{e} \frac{\omega^{2}}{k_{z}^{2}} \frac{\partial f_{0 z}}{\partial v_{z}}\right|_{w / k_{z}} .
$$

Таким образом, и в рассматриваемом случае поглощения геликона, так же как и в случае медленной моды, парциальный коэффициент диффузии в пространстве скоростей может быть найден из анализа парциальной компоненты $Q\left(k_{z}\right)$ энерговыделения пучка волн и не требует расчета пространственного распределения электрических полей. Это обстоятельство позволяет ограничиться при анализе эффективности генерации безындукционного тока и его профиля рассмотрением поведения лучевых траекторий волн и поглощения энергии вдоль них.

Рассмотрено взаимодействие электронов с электромагнитными волнами промежуточного диапазона частот (геликонами) по резонансному механизму Ландау. В результате получено квазилинейное уравнение, которое позволяет описать эволюцию функции распределения электронов и генерацию токов увлечения при нагреве плазмы геликонами. Впервые показано, что, если в анализируемом случае функция распределения факторизуется, оставаясь максвелловской по поперечным скоростям, квазилинейное уравнение сводится к одномерному в пространстве продольных скоростей электронов. Коэффициент диффузии этого одномерного уравнения (11) пропорционален удельной мощности, поглощаемой при взаимодействии частиц с волнами (10).

\section{Финансирование работы}

Вывод полного квазилинейного уравнения для функции распределения в случае поглощения геликона поддержан в рамках государственного задания ФТИ им. А.Ф. Иоффе 0040-2019-0023, уравнение для одномерной функции распределения электронов получено в рамках государственного задания 0034-2021-0002.

\section{Конфликт интересов}

Авторы заявляют, что у них нет конфликта интересов.

\section{Список литературы}

[1] V.L. Vdovin, Plasma Phys. Rep., 39, 95 (2013). DOI: $10.1134 / \mathrm{S} 1063780 \mathrm{X} 13020037$

[2] А.И. Ахиезер, И.А. Ахиезер, Р.В. Половин, А.Г. Ситенко, К.Н. Степанов, Электродинамика плазмы (Наука, М., 1974).

[3] N.J. Fisch, J.M. Rax, Phys. Rev. Lett., 69, 612 (1992). DOI: 10.1103/PhysRevLett.69.612

[4] C.F.F. Karney, N.J. Fisch, Nucl. Fusion, 21, 1549 (1981). DOI: $10.1088 / 0029-5515 / 21 / 12 / 004$

[5] N.J. Fisch, Rev. Mod. Phys., 59, 175 (1981). DOI: $10.1103 /$ RevModPhys.59.175

[6] A.D. Piliya, A.N. Saveliev, Preprint JET-R(98) 01 (JET Joint Undertaking, Abingdon, 1998).

[7] T.H. Stix, Waves in plasmas (American Institute of Physics, N.Y., 1992). 